EXTENDED REPORT

\title{
Outcomes of bleb excision and conjunctival advancement for leaking or hypotonous eyes after glaucoma filtering surgery
}

\author{
D P Tannenbaum, D Hoffman, M J Greaney, J Caprioli
}

See end of article for authors' affiliations

\section{Correspondence to:}

Correspondence to:
Dana P Tannenbaum, MD, Glaucoma Division, Jules Stein Eye Institute, UCLA Medical School, Los Angeles, CA 90095-7000, USA; tannenbaum@ jsei.ucla.edu

Accepted for publication 23 April 2003

\begin{abstract}
Aim: To determine outcomes of bleb excision and conjunctival advancement for leaking or hypotonous eyes after glaucoma filtering surgery.

Methods: In a retrospective case series the medical records of all patients who underwent bleb excision and conjunctival advancement surgery for bleb leaks or hypotony (defined as IOP less than $6 \mathrm{~mm} \mathrm{Hg}$, associated with reduced vision) at the Jules Stein Eye Institute and the Yale University Eye Center between January 1993 and June 2002 were reviewed. Only patients with more than 6 months of follow up were included. Complete success was defined as resolution of the pre-existing leak or hypotony, with maintenance of $I O P \geqslant 6$ and $\leqslant 21 \mathrm{~mm} \mathrm{Hg}$, in the absence of further glaucoma surgery and without any glaucoma medication. Qualified success met the above criteria with or without the use of glaucoma medications. Success rates were analysed with Kaplan-Meier survival curves.

Results: 49 eyes were included in the study; 13 eyes had overt bleb leaks, 27 eyes had hypotony. Nineteen patients underwent resuturing of sclera and/or placement of a pericardial graft in addition to bleb excision and conjunctival advancement. Mean preoperative IOP increased from 3.2 (SD 2.4) mm Hg (range 0$10 \mathrm{~mm} \mathrm{Hg}$ ) to $13.8(4.8) \mathrm{mm} \mathrm{Hg}$ (range $6-29 \mathrm{~mm} \mathrm{Hg}$ ) at last follow up $(\mathrm{p}<0.0001)$. Mean preoperative visual acuity improved from $0.6(0.3)$ logMAR (range 0-2) to $0.3(0.3)$ logMAR (range 0-2) at final follow up $(p<0.0001) .16$ eyes $(40 \%)$ were classified as complete success and 17 additional eyes achieved qualified success, for a total of 33 eyes (83\%) achieving at least qualified success. There was no significant difference in the rate of complete success $(p=0.95)$ or qualified success $(p=0.88)$ between the group of patients who underwent bleb excision and conjunctival advancement alone versus those who also had resuturing of sclera and/or placement of a pericardial graft. Failures included three eyes that had persistent hypotony without leak, requiring additional bleb revision, and four with uncontrolled IOP, two of whom underwent additional glaucoma surgery.

Conclusion: Bleb excision with conjunctival advancement is a successful procedure for bleb repair. This technique provides successful resolution of leaks and hypotony, maintains glaucoma control with or without medication, and preserves vision with minimal postoperative complications in a majority of eyes.
\end{abstract}

$\mathrm{T}$ he introduction of antifibrotic agents as an adjunct to trabeculectomy has resulted in lower postoperative pressures, but at the price of a concomitant increase in vision threatening complications, such as bleb leaks and hypotony. ${ }^{1-9}$

Ideal treatment aims to eliminate leaks and hypotony while preserving filtration function and maintaining target intraocular pressure (IOP). Many non-surgical treatments have been described to address these problems, ${ }^{10-21}$ but successful resolution often requires surgical revision. Various surgical approaches have been reported with varying degrees of success, including conjunctival advancement with or without excision of the pre-existing bleb, ${ }^{52-25}$ free conjunctival grafts, ${ }^{26-28}$ and amniotic membrane grafts. ${ }^{29}$

We have previously described a method for bleb revision that involves excision of the leaking or hypotonous bleb with conjunctival advancement of bulbar conjunctiva from the superior aspect of the globe. ${ }^{22}$ In this study we report a consecutive series of 40 patients who underwent surgical bleb revision by one surgeon (JC) between January 1993 and June 2002 at the Jules Stein Eye Institute and the Yale University Eye Center. Only patients undergoing surgical revision for a bleb leak or hypotony (defined as IOP less than $6 \mathrm{~mm} \mathrm{Hg}$, associated with reduced vision) and who had greater than 6 months follow up were included. Patients who had previously undergone surgical bleb revision were excluded.

\section{METHODS}

The surgical technique for bleb revision was as follows. After anaesthesia was obtained with a retrobulbar injection of $4 \mathrm{ml}$ $2 \%$ lidocaine, a $6 / 0$ silk traction suture was placed in the cornea at the 12 o'clock position to rotate the globe inferiorly. A large conjunctival peritomy was performed extending posteriorly around the bleb from the 9 o'clock to the 3 o'clock position (fig 1). The bleb was then dissected forward, away from the underlying sclera and excised flush with the limbus. All epithelium was removed from the limbus by scraping with a No 67 blade to allow better adhesion of the conjunctiva. The healthy conjunctiva and Tenon's capsule were undermined superiorly. Fibrous attachments between Tenon's capsule and the superior rectus muscle were carefully divided. The conjunctival dissection was limited to the bulbar conjunctiva to avoid trauma to the levator palpebrae muscle. Horizontal conjunctival relaxing incisions were created at the 3 and 9 o'clock positions to allow mobilisation of the tissue.

If leakage of aqueous humour through the sclerostomy was excessive or had caused noticeable shallowing of the anterior chamber during the procedure, the scleral flap was resutured with 10/0 Nylon. Alternatively, if the sclera was friable or the opening into the anterior chamber was large or unprotected, a graft of processed human pericardium (Tutoplast, Innovative Ophthalmic Products Inc, Costa Mesa, CA, USA) was sutured over the site of leakage. A linear partial thickness 


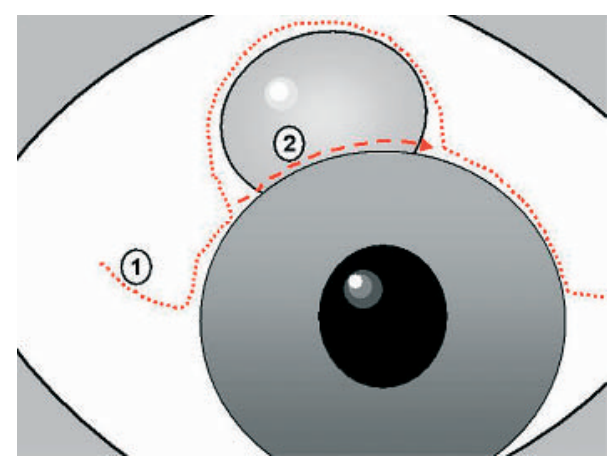

Figure 1 The conjunctival incisions (1, 2) used for bleb revision.

keratotomy was first fashioned immediately posterior to the limbus in the coronal plane (figs 2,3 ). The edge of the pericardium graft was tucked into the pocket created to prevent it from migrating forward onto the cornea and to ensure that draining aqueous was directed posteriorly. It was sutured to the limbus by a 10/0 Nylon horizontal mattress suture. In addition, single 9/0 Vicryl sutures were placed at each of the four corners (fig 2). When necessary, a paracentesis was created with a 25 gauge needle and balanced salt solution was infused through a 27 gauge cannula to deepen the anterior chamber.

The conjunctiva was advanced to the limbus without tension. The relaxing incisions were closed with 9/0 Vicryl sutures and were anchored at the limbus by incorporating a scleral bite (fig 4). Care was taken to ensure that the conjunctiva was relatively taut across the limbus superiorly but not excessively hooded over the cornea. "Dog ears" at the 3 and 9 o'clock positions were excised when suturing the conjunctiva. No suture was placed at the limbus superiorly and no antimetabolite was applied. Antibiotic, steroid, and cycloplegic eye drops were administered postoperatively.

Chart information was abstracted for 40 eyes of 40 patients, including diagnosis, demographic information, type and date of original glaucoma surgery, use of antifibrotic agents, indications for bleb revision, and previous management of the bleb problem. Operative and postoperative courses were reviewed with documentation of visual acuity, intraocular pressure, complications, and the need for glaucoma medications.

Complete success was defined as resolution of the preexisting leak or hypotony, with maintenance of IOP $\geqslant 6 \mathrm{~mm} \mathrm{Hg}$ and $\leqslant 21 \mathrm{~mm} \mathrm{Hg}$, in the absence of further glaucoma surgery and without any glaucoma medication. Qualified success was defined as resolution of the pre-

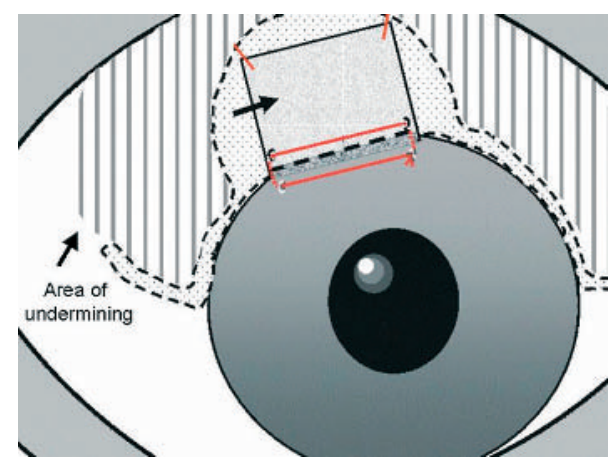

Figure 2 Conjunctiva is dissected (broken line) and pericardium (arrow) is secured by sutures (heavy lines) into a partial keratotomy (lower grey area of pericardium).
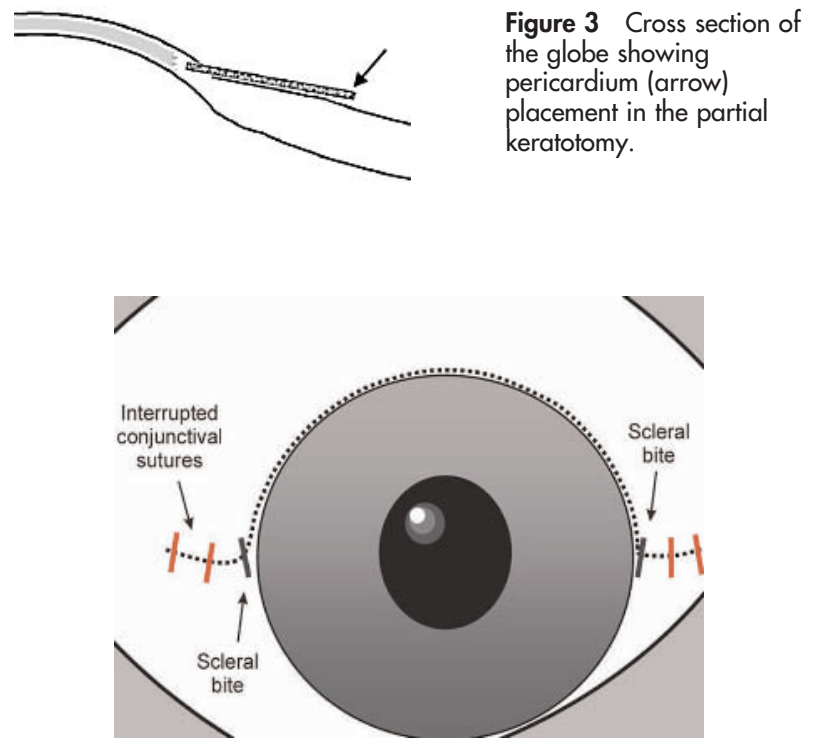

Figure 4 Conjunctiva is closed by suturing (heavy lines) the radial relaxing incisions.

existing leak or hypotony, with maintenance of IOP $\geqslant 6 \mathrm{~mm} \mathrm{Hg}$ and $\leqslant 21 \mathrm{~mm} \mathrm{Hg}$, in the absence of further glaucoma surgery, with or without the use of glaucoma medication. The date of failure was considered the first visit in which recurrence or persistence of the leak or uncontrolled IOP was noted. Success rates were analysed with KaplanMeier survival curves.

\section{RESULTS}

The study group consisted of 19 men and 21 women who ranged in age from 22 to 93 years (mean 63 (SD 15) years). Of the 40 patients, 30 had primary open angle glaucoma, three had normal tension glaucoma, one had pigmentary glaucoma, one had pseudoexfoliative glaucoma, one had juvenile open angle glaucoma, one had angle recession glaucoma, one had glaucoma associated with Axenfeld-Rieger syndrome, and two had chronic angle closure glaucoma. Demographic and clinical characteristics of patients are shown in table 1.

Bleb repair was preceded by trabeculectomy in 37 cases and combined trabeculectomy and cataract extraction in three cases. Adjuvant antifibrotic agents were used intraoperatively in 36 of 40 eyes $(90 \%), 32$ of which had mitomycin C and four of which had 5-fluorouracil. The mean interval between the original trabeculectomy and the decision to repair the bleb was 28.8 (SD 27.0) months (range 0-85 months).

Indications for bleb revision and preoperative examination findings are shown in table 2 . Bleb revision was performed on 13 patients with bleb leaks and 27 patients with hypotony. In nine patients, conservative therapies were attempted before surgical revision; Nd:YAG laser (one case), mattress sutures (three cases), intrableb autologous blood injection (five cases), cryotherapy (one case), trichloroacetic acid (one case), cautery (one case). Three patients each underwent two non-incisional procedures.

At the time of bleb revision, the flow of aqueous through the sclerostomy was deemed excessive in 19 (48\%) cases. Processed human pericardium was sutured in place in 13 (33\%) of these and the scleral flap was resutured in nine $(23 \%)$, with three patients having both procedures. One of the patients who underwent resuturing of the scleral flap, also required drainage of choroidal effusions. These procedures were performed in addition to bleb excision and conjunctival advancement. 


\begin{tabular}{ll}
\hline Table 1 Demographics and clinical characteristics \\
\hline Characteristic & No of patients (total 40) \\
\hline Mean age (SD, range) (years) & $63(15,22-93)$ \\
Male:female ratio & $19: 21$ \\
Diagnosis & 30 \\
Primary open angle glaucoma & 3 \\
Normal tension glaucoma & 1 \\
Pseudoexfoliative glaucoma & 1 \\
Pigmentary glaucoma & 1 \\
Juvenile open angle glaucoma & 1 \\
Axenfeld-Rieger syndrome & 1 \\
Angle recession glaucoma & 2 \\
Chronic angle closure glaucoma & \\
Type of previous glaucoma procedure & 37 \\
Trabeculectomy & 3 \\
Combined surgery & 32 \\
Antifibrotic agent & 4 \\
Mitomycin C & 4 \\
5-Fluorouracil & \\
None &
\end{tabular}

Outcomes are shown in table 3. The mean follow up period after surgical bleb revision was 22.3 (17.9) months (range 684 months). The mean preoperative IOP was 3.2 (2.4) $\mathrm{mm} \mathrm{Hg}$ (range $0-10 \mathrm{~mm} \mathrm{Hg}$ ), and the mean postoperative IOP at last follow up was 13.8 (4.8) mm Hg (range $6-29 \mathrm{~mm} \mathrm{Hg})(\mathrm{p}<0.0001)$. A scatter plot of mean preoperative and final IOP is shown in figure 5. On postoperative day 1 the mean IOP for patients who had concurrent placement of pericardial grafts and/or resuturing of the scleral flap $(n=19)$ was $16.4(12.9) \mathrm{mm} \mathrm{Hg}$; the mean IOP of the patients who had bleb excision and conjunctival advancement alone $(\mathrm{n}=21)$ was $11.2(14.9) \mathrm{mm} \mathrm{Hg}(\mathrm{p}=0.25)$. The mean postoperative number of medications per patient was 0.7 (0.9) (range $0-4$ ). The mean preoperative visual acuity was 0.6 (0.3) logMAR (range 0-2 logMAR), and the mean postoperative visual acuity at final examination was $0.3(0.3)$ $\log$ MAR (range $0-2 \log$ MAR) $(\mathrm{p}<0.0001)$. At the last follow up visit, the visual acuity had improved in 34 patients, was the same in two patients, and had worsened in four patients. Deterioration of visual acuity was determined to be secondary to worsening cataracts in three cases. One patient subsequently underwent penetrating keratoplasty with poor visual outcome.

Complete success was obtained in 16 of 40 patients (40\%) in providing resolution of the pre-existing leak or hypotony. Qualified success was obtained in an additional 17 patients, for a total of 33 eyes (83\%) achieving at least qualified success. In all 13 cases in which a bleb leak was present, the leak was successfully repaired by a single procedure. Three eyes had persistent hypotony without leak and required additional bleb revision. Three patients had IOP $>21 \mathrm{~mm} \mathrm{Hg}$ despite glaucoma medications, and one of these had subsequent placement of an Ahmed valve. One additional patient underwent placement of an Ahmed valve for IOP control, although IOP remained $<21 \mathrm{~mm} \mathrm{Hg}$ throughout the entire follow up period. Of the seven patients who failed bleb revision, the primary surgery in six of the cases was trabeculectomy with MMC. In one failure, no antimetabolite was used in the original trabeculectomy. Figure 6 shows the survival curves for complete and qualified success.

In the subset of patients who underwent resuturing of sclera and/or placement of a pericardial graft in addition to bleb excision and conjunctival advancement, 16 of the 19 (84\%) obtained at least qualified success and eight of 19 $(42 \%)$ obtained complete success. There was no significant difference in the rate of complete success $(p=0.95)$ or
Table 2 Indications for bleb revision and preoperative findings

\begin{tabular}{ll}
\hline & No of patients \\
\hline Indications & 13 \\
Bleb leak & 27 \\
Hypotony & \\
Findings & 4 \\
Blebitis & 16 \\
Macular folds & 11 \\
Choroidal effusion & 1 \\
Corneal oedema & \\
\hline
\end{tabular}

qualified success $(p=0.88)$ between this subset of patients and the group of patients who underwent bleb excision and conjunctival advancement alone. Of the two failures that required placement of an Ahmed valve, one patient had undergone resuturing of the scleral flap and placement of a pericardial graft at the time of bleb excision and conjunctival advancement, while the second patient did not.

Complications from the bleb revision included one patient who had an IOP spike on the first postoperative day. There were no cases of immediate or late postoperative bleb related infections, suprachoroidal haemorrhage, choroidal effusions, or shallow anterior chambers after bleb revision.

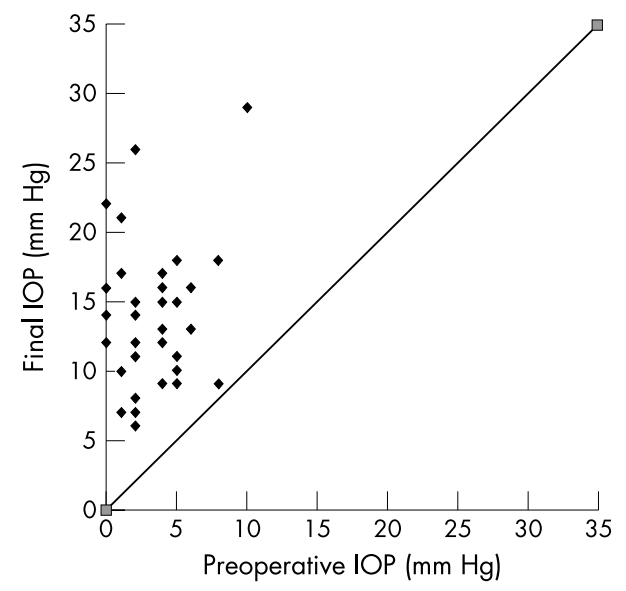

Figure 5 Scatter plot of preoperative and final IOP. 


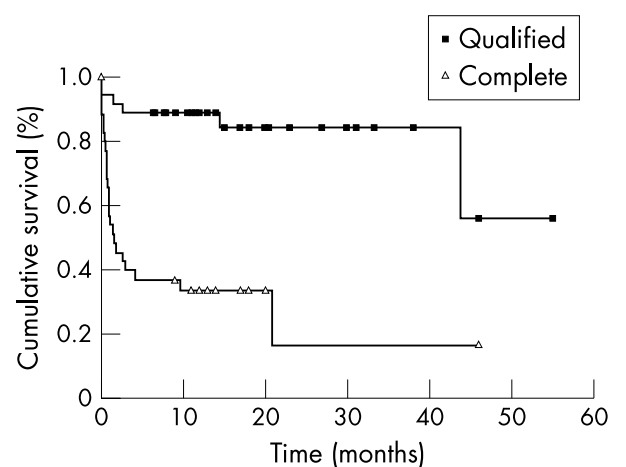

Figure 6 Complete and qualified success, Kaplan-Meier survival curves.

\section{DISCUSSION}

Antifibrotic agents have been shown to be associated with a higher incidence and prevalence of bleb leaks and hypotony. ${ }^{1-9}$ A variety of techniques have been documented for their treatment, including pharmacological aqueous suppression, ${ }^{10}$ tamponade with an eye patch or oversized contact lens, ${ }^{11-13}$ subconjunctival injection of autologous blood, ${ }^{14-17}$ application of cyanoacrylate tissue adhesive, ${ }^{10}{ }^{14}$ trichloroacetic acid surface abrasion, ${ }^{19}$ and treatment with Nd:YAG laser $^{20}$ and argon laser. ${ }^{21}$ Each of these methods has met with limited success. Additionally, many of these studies predate the use of antifibrotic agents and do not report on long term IOP control.

Surgical bleb revision has become increasingly necessary for management of trabeculectomy complications in the era of antifibrotic agents. Conjunctival advancement with or without bleb excision, ${ }^{52-25}$ free conjunctival autografts, ${ }^{26-29}$ and amniotic membrane grafts ${ }^{30}$ have been reported with varying degrees of success.

Our technique of bleb revision with conjunctival advancement is relatively simple. After complete excision of the bleb, extensive undermining of the superior conjunctiva and Tenon's capsule is performed with dissection of attachments around the superior rectus muscle. Tension is eliminated by the creation of conjunctival relaxing incisions that are then closed with $9 / 0$ Vicryl sutures and anchored to the limbus by incorporating a scleral bite. Watertight adhesion and subsequent healing of the conjunctiva is ensured by denudation of all epithelium from the limbal region. Our technique differs from other methods of conjunctival advancement in which the pre-existing filtering bleb is left intact. ${ }^{25}{ }^{31}$ Other differences include the use of relaxing incisions and no need for the placement of a mattress suture ${ }^{50}$ or running suture ${ }^{23}$ at the limbus.

It is our opinion that conjunctival advancement with excision of the pre-existing bleb is superior to conjunctival advancement alone. We speculate that the presence of the pre-existing bleb leads to an increased incidence of recurrent bleb leaks as a result of retained epithelial inclusions, as well as to the development of bleb dysaesthesia.

In our study, bleb excision with conjunctival advancement eliminated leaks and hypotony in 37 of 40 patients (93\%). Three patients $(8 \%)$ required repeat bleb revision for persistent hypotony; two patients within 2 months and one patient 18 months after initial bleb revision. No patients had persistence or recurrence of bleb leak, a frequent complication reported in other studies. ${ }^{5} 23252728$

Catoira $e t a l^{25}$ reported a series of 30 eyes that underwent conjunctival advancement with preservation of the preexisting bleb for repair of bleb leaks, hypotony, and dysaesthesia. A 10\% incidence of recurrent bleb leaks and a
$7 \%$ incidence of persistent hypotony were reported. In a separate study comparing surgical bleb revision versus nonincisional treatment for bleb leaks, Burnstein and colleagues $^{31}$ reported a group of 34 eyes that underwent advancement of conjunctiva over surgically de-epithelialised blebs. They found a $6 \%$ incidence of recurrent bleb leaks and a $6 \%$ incidence of bleb dysaesthesia.

We believe that bleb excision with evaluation of the underlying scleral flap is a critical step in bleb revision, particularly in patients who demonstrate hypotony in the absence of a frank conjunctival leak. This allows for placement of additional scleral sutures or a pericardial graft to secure an overfiltering or fragmented flap.

Bleb revision with placement of additional scleral sutures to secure an overfiltering but intact flap has been well documented. $^{32}$ Suner et $a l^{33}$ evaluated a technique for elimination of hypotony, in the absence of a leak, by reoperation using two sets of stitches in the scleral flap, with one set tied tightly to temporarily raise the IOP, stretch the sclera, and flatten choroidal folds. Restoration of prefiltration visual acuity without compromise of bleb function was reported. In addition, heterologous sclera, heterologous pericardium, and autologous Tenon's capsule have all been used to restrict aqueous outflow when the integrity of the scleral flap is compromised..$^{34}$ Nineteen of 40 patients (48\%) in our study underwent resuturing of sclera and/or placement of a pericardial graft, in addition to bleb excision and conjunctival advancement. On postoperative day 1 the mean IOP for patients in this group was higher than the mean IOP for patients who underwent bleb excision and conjunctival advancement alone but not significantly $(p=0.25)$. There was, however, no significant difference in the rate of complete success $(p=0.91)$ or qualified success $(p=0.85)$ between these two groups. Placement of additional scleral sutures or a pericardial graft at the time of bleb revision does not adversely affect long term IOP control and may decrease the incidence of recurrent hypotony in the postoperative period.

There is a justifiable concern that a leaking filtering bleb may be transformed into a non-functioning filtering bleb when a conjunctival advancement flap is performed. In our study, 17 of 40 patients (43\%) required glaucoma medications to maintain adequate control of IOP. Two patients (5\%) required additional glaucoma surgery with placement of an Ahmed valve; this occurred 13 months after the initial bleb revision in one patient and after 48 months in the other. The percentage of patients requiring glaucoma medications after surgery in this case series was less than that reported in other studies using a similar technique. ${ }^{523}$

Budenz et $a l^{5}$ described bleb excision with conjunctival advancement for late onset bleb leaks in 26 eyes. Resolution of the leak was achieved in 24 of 26 patients (92\%); however, 13 patients $(50 \%)$ required additional glaucoma medications and two patients ( $8 \%$ ) required further glaucoma surgery for IOP control. In a second study by Myers and associates, ${ }^{23} 16$ patients underwent bleb excision with conjunctival advancement for repair of bleb leaks and hypotony. This technique was successful in closing the leak and increasing the IOP in 15 of 16 cases. In this series, 10 of the 16 patients (63\%) required additional glaucoma medications to achieve adequate IOP control. The fact that most of the patients in this study $(69 \%)$ did not have adjunctive antimetabolites at the time of the original surgery may have influenced the outcome of bleb revision.

The most frequent complication in our series was persistent hypotony in the early postoperative period, for which three patients underwent repeat bleb revisions. One patient developed an IOP spike on postoperative day 1 . Finally, the late complication of bleb failure requiring additional filtering 
surgery occurred twice in our series. No patients experienced clinically or cosmetically significant ptosis or hyperopia, complications which have been reported subsequent to conjunctival advancement. ${ }^{52}$ It has been postulated that these complications are secondary to shortening of the superior conjunctiva or inferior traction on the conjunctival attachments of the superior rectus. ${ }^{25}$ The meticulous conjunctival dissection around the superior rectus and the conjunctival relaxing incisions in our technique decreases the risk of such complications.

Bleb excision with conjunctival advancement is a successful procedure for bleb repair, thereby reducing the risk of vision threatening sequelae. This technique provides successful resolution of leaks and hypotony, maintains glaucoma control with or without medication, and preserves vision with minimal postoperative complications in a majority of eyes.

\section{ACKNOWLEDGEMENTS}

Grant support from Research to Prevent Blindness Inc, New York, USA (JC); the Glaucoma Research Foundation (JC); TFC Frost Charitable Fund, UK (MJG); British Medical Association (Nathaniel Bishop Harman Award), UK (MJG); and the Fulbright Commission, UK (MJG).

\section{Authors' affiliations}

D P Tannenbaum, D Hoffman, J Caprioli, Jules Stein Eye Institute, UCLA Medical School, Los Angeles, CA, USA

M J Greaney, Bristol Eye Hospital, Lower Maudlin Street, Bristol BS1 2LX, UK

Proprietary interest: None.

Commercial interest: None.

\section{REFERENCES}

1 Greenfield DS, Liebmann JM, Jee J, et al. Late-onset bleb leaks after glaucoma filtering surgery. Arch Ophthalmol 1998;116:443-7.

2 Ticho U, Ophir A. Late complications after glaucoma filtering surgery with adjunctive 5-fluorouracil. Am J Ophthalmol 1993;1 15:506-10.

3 Greenfield DS, Suner IJ, Miller MP, et al. Endophthalmitis after filtering surgery with Mitomycin. Arch Ophthalmol 1996;114:943-9.

4 Soltau JB, Rothman RF, Budenz DL, et al. Risk factors for glaucoma filtering bleb infections [see comments]. Arch Ophthalmol 2000; 1 18:338-42.

5 Budenz DL, Chen PP, Weaver YK. Conjunctival advancement for late-onset filtering bleb leaks: indications and outcomes. Arch Ophthalmol 1999.117:1014-19.

6 Waheed S, Ritterband DC, Greenfield DS, et al. Bleb-related ocular infection in children after trabeculectomy with Mitomycin C. Ophthalmology 1997:104:2117-20.

7 Stamper RL, McMenemy MG, Lieberman MF. Hypotonous maculopathy after trabeculectomy with subconjunctival 5-fluorouracil. Am J Ophthalmol $1992 ; 114: 544-53$.
8 Costa VP, Wilson RP, Moster MR, et al. Hypotony maculopathy following the use of topical mitomycin $C$ in glaucoma filtration surgery. Ophthalmic Surg 1993;24:389-94

9 Zacharia PT, Deppermann SR, Schuman JS. Ocular hypotony after trabeculectomy with Mitomycin C. Am J Ophthalmol 1993;116:314-26.

10 Asrani SG, Wilensky JT. Management of bleb leaks after glaucoma filtering surgery. Use of autologous fibrin tissue glue as an alternative. Ophthalmology 1996; 103:294-8

11 Ruderman JM, Allen RC. Simmons' tamponade shell for leaking filtration blebs. Arch Ophthalmol 1985;103:1708-10.

12 Blok MD, Kok JH, van Mil C, et al. Use of the megasoft bandage lens for treatment of complications after trabeculectomy. Am J Ophthalmol 1990; 110:264-8

13 Fourman S, Wiley L. Use of a collagen shield to treat a glaucoma filter bleb leak. Am J Ophthalmol 1989;107:673-4.

14 Smith MF, Magauran RGr, Betchkal J, et al. Treatment of postfiltration bleb leaks with autologous blood. Ophthalmology 1995;102:868-71.

15 Choudhri SA, Herndon LW, Damii KF, et al. Efficacy of autologous blood injection for treating overfiltering or leaking blebs after glaucoma surgery. Am J Ophthalmol 1997;123:554-5.

16 Leen MM, Moster MR, Katz $\sqcup$, et al. Management of overfiltering and leaking blebs with autologous blood injection. Arch Ophthalmol 1995; 13:1050-5.

17 Wise JB. Treatment of chronic postfiltration hypotony by intrableb injection of autologous blood. Arch Ophthalmol 1993;111:827-30.

18 Bettin P, Carassa RG, Fiori $M$, et al. Treatment of hyperfiltering blebs with $\mathrm{Nd}$ :YAG laser-induced subconjunctival bleeding. J Glaucoma 1999;8:380-3.

19 Gehring JR, Ciccarelli EC. Trichloracetic acid treatment of filtering blebs following cataract extraction. Am J Ophthalmol 1972;74:622-4.

20 Geyer 0 . Management of large, leaking, and inadvertent filtering blebs with the neodymium:YAG laser. Ophthalmology 1998;105:983-7.

21 Hennis HL, Stewart WC. Use of the argon laser to close filtering bleb leaks. Graefes Arch Clin Exp Ophthalmol 1992;230:537-541.

22 O'Conner DJ, Tressler CS, Caprioli J. A surgical method to repair leaking filtering blebs. Ophthalmic Surg 1992;23:336-338.25.

23 Myers JS, Yang CB, Herndon LW, et al. Excisional bleb revision to correct over filtration or leakage. J Glaucoma 2000;9:169-73.

24 Wadhwani RA, Bellows AR, Hutchinson BT. Surgical repair of leaking filtering blebs. Ophthalmology 2000;107:1681-7.

25 Catoira Y, Wudunn D, Cantor LB. Revision of dysfunctional filtering blebs by conjunctival advancement with bleb preservation. Am J Ophthalmol 2000;130:574-9

26 Wilson MR, Kotas-Neumann R. Free conjunctival patch for repair of persistent late bleb leak. Am J Ophthalmol 1994; 17:569-74.

27 Sugar HS. Complications, repair and reoperation of antiglaucoma filtering blebs. Am J Ophthalmol 1967;63:825-33.

28 Harris LD Yang G, Feldman RM, et al. Autologous conjunctival resurfacing of leaking filtering blebs. Ophthalmology 2000;107:1676-80.

29 Schnyder CC, Shaarawy T, Ravinet E, et al. Free conjunctival autologous graft for bleb repair and reduction after trabeculectomy and nonpenetrating filtering surgery. J Glaucoma 2002;11:10-16.

30 Budenz DL, Barton K, Tseng SC. Amniotic membrane transplantation for repair of leaking glaucoma filtering blebs. Am J Ophthalmol 2000;130:580-8.

31 Burnstein AL, WuDunn D, Knotts SL, et al. Conjunctival advancement versus nonincisional treatment for late-onset glaucoma filtering bleb leaks. Ophthalmology 2002;109:71-5.

32 Galin MA, Hung PT. Surgical repair of leaking blebs. Am J Ophthalmol 1977;83:328-33

33 Suner IJ, Greenfield DS, Miller MP, et al. Hypotony maculopathy after filtering surgery with Mitomycin C. Incidence and treatment [see comments]. Ophthalmology 1997; 104:207-14, discussion 14-5.

34 La Borwit SE, Quigley HA, Jampel HD. Bleb reduction and bleb repair after trabeculectomy. Ophthalmology 2000;107:712-18. 